\title{
Phase separation effects and the nematic-isotropic transition in polymer and low molecular weight liquid crystals doped with nanoparticles
}

\author{
Maxim V. Gorkunov, ${ }^{* a}$ Georgiy A. Shandryuk, Alina M. Shatalova, \\ Irina Yu. Kutergina, ${ }^{b}$ Alexey S. Merekalov, ${ }^{b}$ Yaroslav V. Kudryavtsev, ${ }^{\mathrm{b}}$ Raisa V. Talroze ${ }^{\mathrm{b}}$ \\ and Mikhail A. Osipov ${ }^{c}$ \\ Properties of the nematic-isotropic phase transition in polymer and low molecular weight liquid crystals \\ doped with nanoparticles have been studied both experimentally and theoretically in terms of \\ molecular mean-field theory. The variation of the transition temperature and the transition heat with \\ the increasing volume fraction of CdSe quantum dot nanoparticles in copolymer and low molecular \\ weight nematics has been investigated experimentally and the data are interpreted using the results of \\ the molecular theory which accounts for a possibility of phase separation when the system undergoes \\ the nematic-isotropic transition. The theory predicts that the nematic and isotropic phases with \\ different concentrations of nanoparticles may coexist over a broad temperature range, but only if the \\ nanoparticle volume fraction exceeds a certain threshold value which depends on the material \\ parameters. Such unusual phase separation effects are determined by the strong interaction between \\ nanoparticles and mesogenic groups and between nanoparticles themselves.
}

Received 25th October 2012

Accepted 30th January 2013

DOI: $10.1039 / \mathrm{c} 3 \mathrm{sm} 27467 \mathrm{e}$

www.rsc.org/softmatter effect. ${ }^{15}$ In contrast, the N-I transition temperature increases in nematics doped with strongly anisotropic NPs including nanotubes, ${ }^{\mathbf{1 6}}$ magnetic nanorods ${ }^{\mathbf{1 7}}$ and various ferroelectric particles. ${ }^{9,10}$ It has also been found that the doping of the classical nematic LC 5CB with plate-like cis isomers of the dye results in a softening of the first-order N-I transition. ${ }^{18}$ All of these effects have been described by a molecular theory developed in our previous paper ${ }^{15}$ and in ref. 19.

Recently cadmium selenide (CdSe) quantum dot NPs have been embedded into nematic, smectic and cholesteric side chain polymer matrices with an ultimate goal to control and improve the photoluminescent properties of quantum dot NPs. ${ }^{20-22}$ In general, polymer LCs doped with various NPs are considered to be promising materials because of their mechanical stability and a possibility to manufacture thin glassy films. The anisotropic structure of a LC polymer may also provide a way to control the arrangement of inorganic NPs within the matrix. ${ }^{20}$ Interactions of the polymer carboxylic groups with the surface of NPs govern the localization of the NPs and result in the formation of polymer nanolayers separated by the nanolayers of NPs.

Minimum size of NPs may be close to molecular dimensions, but the properties of the particles differ significantly from those of surrounding mesogenic molecules and therefore a phase separation should be a common phenomenon. The simplest phase separation between NPs and mesogenic molecules may occur already in the isotropic phase and is not related to the 
nematic ordering. Such a demixing, however, can be successfully suppressed by attaching appropriate organic groups to the surface of the NP which makes it more compatible with the surrounding LC medium.

At the same time, there exists a different type of phase separation which is accompanied by the transition into the nematic phase, and which is more difficult to control. The N-I transition temperature decreases with the increasing concentration of NPs and as a result the nematic phase is partially destabilized. The total free energy may be minimized if the system separates into the isotropic phase with an increased concentration of NPs and the nematic phase with lower concentration of NPs. This kind of phase separation is very important for the application of LC nanocomposites, and it has been observed in few anisotropic soft matter systems. ${ }^{23,24}$ However, it has not been studied in detail so far neither experimentally nor theoretically. One notes also that phase separation in LCs doped with NPs is not always a negative effect which should be avoided. For example, in LC blue phases NPs mainly concentrate in the vicinity of defect cores which results in a decrease of the energy of these defects thus stabilizing the blue phases..$^{25}$ Very recently it has been shown that gold NPs with mesogenic coatings form reversible networks composed of nematic droplets accompanied by disclination lines and loops by enrichment of the NPs at the nematic-isotropic liquid interfaces.

It is well known that a similar phase separation occurs in mixtures of LCs and, in particular, in nematics doped with nonmesogenic molecules (see e.g. ref. 26 and 27) although the corresponding two-phase region around the N-I transition is usually rather narrow. Such a phase separation in nematic LCs has been described theoretically in ref. 28. One notes, however, that the results of the molecular theory presented in ref. 28 cannot be used to describe the effect of NPs on the N-I transition because the theory is based on the strong assumption that the chemical potentials of the two compounds are independent of their concentrations in the isotropic phase. This assumption is only valid if the properties of the dopant are very similar to those of the host which is generally not valid for typical NPs that are very different from mesogenic molecules. Very recently a simple mean-field theory of LCs doped with colloidal particles has been developed in ref. 29 and 30 where the phase separation effects have been taken into account. In these papers, however, the phase separation is not studied in detail and the interaction energy of the mixture is oversimplified which may be justified in the case of colloids but not in mixtures of mesogenic molecules and small NPs.

In this paper we develop a general molecular theory of phase separation in nematic LCs doped with NPs and show that, depending on the relative strength of interaction between NPs and mesogenic molecules, the nematic and the isotropic phases may coexist over a very broad temperature interval. In this case the system undergoes a transition into the low temperature smectic or crystal phase remaining in the phase-separated state. We have studied experimentally the properties of the N-I phase transition in composites for different values of the volume fraction of NPs. An unusual behavior of the transition temperature and the transition heat has been found which can be interpreted under the assumption that the nematic and isotropic phases with different concentrations of NPs may coexist over a very broad temperature interval. The same measurements have been repeated for a low molecular weight (LMW) LC material in order to confirm that this behavior is not specific for polymer systems but should be a general feature of anisotropic soft matter materials.

\section{Molecular theory of phase separation in polymer nematics doped with nanoparticles}

\subsection{Free energy of the nematic phase}

Consider the side chain polymer nematic LC doped with spherical NPs. We introduce the number densities $\rho_{\mathrm{m}}, \rho_{\mathrm{n}}, \rho_{\mathrm{s}}$ and $\rho_{\text {ch }}$ of mesogenic groups, NPs, weakly anisotropic segments of the main chain and polymer macromolecules, respectively. Everywhere below the subscript $\mathrm{n}$ denotes NPs, $\mathrm{m}$ denotes mesogenic molecules, s denotes segments of the main chains and ch denotes main chains. In the consistent theory of such a system one has to take into account both isotropic and anisotropic interactions between mesogenic groups, NPs and segments of the main polymer chain. As a result in any fluid phase the system is characterized by the following total intermolecular interaction potential integrated over all intermolecular vectors:

$$
\begin{aligned}
H= & -\frac{1}{2 V} \sum_{i j}\left[U_{\mathrm{mm}}\left(\boldsymbol{a}_{i} \cdot \boldsymbol{a}_{j}\right)+U_{\mathrm{ms}}\left(\boldsymbol{a}_{i} \cdot \boldsymbol{a}_{j}\right)+U_{\mathrm{ss}}\left(\boldsymbol{a}_{i} \cdot \boldsymbol{a}_{j}\right)+U_{\mathrm{nn}}\right. \\
& \left.+U_{\mathrm{nm}}+U_{\mathrm{ns}}\right]+\frac{1}{V} \sum_{i} \Phi\left(\boldsymbol{a}_{i} \cdot \boldsymbol{a}_{i+1}, \boldsymbol{r}_{i, i+1}\right),
\end{aligned}
$$

where $V$ is the system volume. Here $U_{\mathrm{mm}}\left(\boldsymbol{a}_{i} \cdot \boldsymbol{a}_{j}\right)$ is the integrated anisotropic interaction potential between mesogenic groups which depends on the unit vectors $\boldsymbol{a}_{i}$ and $\boldsymbol{a}_{j}$ in the direction of the long molecular axes of the groups $i$ and $j$, respectively. $U_{\mathrm{ms}}\left(\boldsymbol{a}_{i} \cdot \boldsymbol{a}_{j}\right)$ is the anisotropic interaction potential between a mesogenic group and a segment of the main chain while $U\left(\boldsymbol{a}_{i} \cdot \boldsymbol{a}_{j}\right)$ is the corresponding interaction energy between segments in different main chains. The constant terms $U_{\mathrm{nn}}+$ $U_{\mathrm{nm}}+U_{\mathrm{ns}}$ describe isotropic interactions between NPs, mesogenic groups and segments of the main chain. Finally, in the last term $\Phi\left(\boldsymbol{a}_{i} \cdot \boldsymbol{a}_{i+1}, \boldsymbol{r}_{i, i+1}\right)$ the anisotropic interaction between neighboring segments of the same main chain which depends on both relative orientation and relative position of the neighboring segments $\boldsymbol{r}_{i, i+1}$.

The functions $U_{\mathrm{mm}}\left(\boldsymbol{a}_{i} \cdot \boldsymbol{a}_{j}\right), U_{\mathrm{ms}}\left(\boldsymbol{a}_{i} \cdot \boldsymbol{a}_{j}\right), U_{\mathrm{ss}}\left(\boldsymbol{a}_{i} \cdot \boldsymbol{a}_{j}\right)$ can be expanded in Legendre polynomials $P_{n}\left(\boldsymbol{a}_{i} \cdot \boldsymbol{a}_{j}\right)$ taking into account the first nonpolar term which is responsible for the nematic ordering:

$$
U\left(\boldsymbol{a}_{i} \cdot \boldsymbol{a}_{j}\right) \approx U_{0}+J P_{2}\left(\boldsymbol{a}_{i} \cdot \boldsymbol{a}_{j}\right) .
$$

Here $P_{2}(x)=3 x^{2} / 2-1 / 3$ is the second Legendre polynomial. One notes that the quantities $U_{\mathrm{pq}}, \mathrm{p}, \mathrm{q}=\mathrm{n}, \mathrm{m}, \mathrm{s}, U_{0}$ and $J$ have the dimension of energy $\times$ volume.

Taking into account the interaction between NPs and the mesogenic groups in the molecular-field approximation the free 
energy can be expressed as $F=U-k T \Sigma-k T V \rho_{\mathrm{ch}} \ln Z$ where $U$ is the average interparticle energy defined as the average Hamiltonian $H$ given by eqn (1) except for the last term which determines the conformational partition function of the single chain $-k T \rho_{\mathrm{ch}} \ln Z .^{31}$ The entropy $\Sigma$ is a sum of the mixing entropy of the NPs and the mesogenic groups and the entropy associated with the orientational ordering of mesogenic groups in the nematic phase.

In the molecular field approximation the average $\left\langle P_{2}\left(\boldsymbol{a}_{i} \cdot \boldsymbol{a}_{j}\right)\right\rangle=S^{2}$, where $S=\left\langle P_{2}\left(\boldsymbol{a}_{i} \cdot \boldsymbol{n}\right)\right\rangle$ is the nematic order parameter and the unit vector $\boldsymbol{n}$ is the nematic director. In the isotropic phase $S=0$ and thus only the isotropic parts of the average interaction potentials contribute to the internal energy $U$. Performing the summation over all pairs of particles the average interparticle energy density in the isotropic phase can be expressed as

$$
U / V=-\frac{1}{2} \rho_{\mathrm{m}}{ }^{2} U_{\mathrm{mm}}-\frac{1}{2} \rho_{\mathrm{s}}{ }^{2} U_{\mathrm{ss}}-\rho_{\mathrm{m}} \rho_{\mathrm{n}} U_{\mathrm{mn}}-\rho_{\mathrm{m}} \rho_{\mathrm{s}} U_{\mathrm{sm}}-\rho_{\mathrm{n}} \rho_{\mathrm{s}} U_{\mathrm{sn}} .
$$

Now the free energy of the isotropic phase of such a composite can be expressed as:

$$
\begin{aligned}
\frac{1}{V} F_{\mathrm{I}}= & k_{\mathrm{B}} T \rho_{\mathrm{n}}\left(\ln \rho_{\mathrm{n}}-1\right)+k_{\mathrm{B}} T \rho_{\mathrm{ch}}\left(\ln \rho_{\mathrm{ch}}-1\right) \\
& -\frac{1}{2} \rho_{\mathrm{n}}^{2} U_{\mathrm{nn}}-\frac{1}{2} \rho_{\mathrm{m}}^{2} U_{\mathrm{mm}}-\frac{1}{2} \rho_{\mathrm{s}}^{2} U_{\mathrm{ss}}-\rho_{\mathrm{m}} \rho_{\mathrm{n}} U_{\mathrm{mn}} \\
& -\rho_{\mathrm{m}} \rho_{\mathrm{s}} U_{\mathrm{sm}}-\rho_{\mathrm{n}} \rho_{\mathrm{s}} U_{\mathrm{sn}}-k_{\mathrm{B}} T \rho_{\mathrm{ch}} \ln Z_{\mathrm{I}}
\end{aligned}
$$

where the first two terms describe the entropy of mixing of NPs and polymer chains, and the last term is the conformational free energy of the main polymer chain, in which the partition function can be written in the form:

$$
Z_{\mathrm{I}}=\int \exp \left[-\beta \sum_{i} \Phi\left(\boldsymbol{a}_{i} \cdot \boldsymbol{a}_{i+1}, \boldsymbol{r}_{i}-\boldsymbol{r}_{i+1}\right)\right] \mathrm{d} \Gamma,
$$

where $\mathrm{d} \Gamma$ denotes the integration over all positions $\boldsymbol{r}_{i}$ and orientations $\boldsymbol{a}_{i}$ of the segments of the chain.

The free energy of the nematic phase includes also the energy terms which depend on the nematic order parameter $S$ determined by the anisotropic part of the intermolecular interactions, and the orientational entropy. Neglecting the weak anisotropic interaction between segments of different chains, the free energy of the nematic phase is given by the following expression:

$$
\begin{aligned}
\frac{1}{V} F_{\mathrm{N}}= & k_{\mathrm{B}} T \rho_{\mathrm{n}}\left(\ln \rho_{\mathrm{n}}-1\right)+k_{\mathrm{B}} T \rho_{\mathrm{ch}}\left(\ln \rho_{\mathrm{ch}}-1\right)-\frac{1}{2} \rho_{\mathrm{n}}{ }^{2} U_{\mathrm{nn}} \\
& -\frac{1}{2} \rho_{\mathrm{m}}{ }^{2} U_{\mathrm{mm}}-\frac{1}{2} \rho_{\mathrm{s}}{ }^{2} U_{\mathrm{ss}}-\rho_{\mathrm{m}} \rho_{\mathrm{n}} U_{\mathrm{mn}}-\rho_{\mathrm{m}} \rho_{\mathrm{s}} U_{\mathrm{sm}}-\rho_{\mathrm{n}} \rho_{\mathrm{s}} U_{\mathrm{sn}} \\
& +\frac{1}{2} \rho_{\mathrm{m}}{ }^{2} J_{\mathrm{mm}} S_{\mathrm{m}}{ }^{2}+\rho_{\mathrm{m}} \rho_{\mathrm{s}} S_{\mathrm{m}} S_{\mathrm{s}}-k_{\mathrm{B}} T \rho_{\mathrm{ch}} \ln Z_{\mathrm{N}} \\
& -k_{\mathrm{B}} T \int f_{\mathrm{m}}(\boldsymbol{a}) \ln f_{\mathrm{m}}(\boldsymbol{a}) \mathrm{d} \boldsymbol{a}
\end{aligned}
$$

where the last term is the orientational entropy of mesogenic groups in the nematic phase, $f_{\mathrm{m}}(\boldsymbol{a})$ is the orientational distribution function of the mesogenic groups and $S_{\mathrm{m}}$ and $S_{\mathrm{s}}$ are the scalar nematic order parameters of mesogenic groups and segments of the main chain, respectively, expressed as:

$$
S_{\mathrm{m}, \mathrm{s}}=\int P_{2}(\boldsymbol{a} \cdot \boldsymbol{n}) f_{\mathrm{m}, \mathrm{s}}(\boldsymbol{a} \cdot \boldsymbol{n}) \mathrm{d} \boldsymbol{a} .
$$

The conformational partition function of the main chain in the nematic phase reads:

$$
Z_{\mathrm{N}}=\int \exp \left\{-\beta \sum_{i}\left[U\left(\boldsymbol{a}_{i}, \boldsymbol{a}_{i+1}, \boldsymbol{r}_{i}-\boldsymbol{r}_{i+1}\right)+U_{\mathrm{MF}}\left(\boldsymbol{a}_{i}\right)\right]\right\} \mathrm{d} \Gamma,
$$

where $U_{\mathrm{MF}}\left(\boldsymbol{a}_{i}\right)$ is the average molecular field acting on the segment $i$ of the main chain:

$$
U_{\mathrm{MF}}\left(\boldsymbol{a}_{i}\right)=J_{\mathrm{ms}} S_{\mathrm{m}} P_{2}\left(\boldsymbol{a}_{i} \cdot \boldsymbol{n}\right)
$$

The full partition function of the polymer chain accounts for chain flexibility and for the effect of the mean field on the energy of the chain. It is determined by the Green function which is defined as a partition function $Z(\Theta, N)$ of the chain with fixed orientation of the last segment, $\cos \left(\boldsymbol{a}_{N} \cdot \boldsymbol{n}\right)=1$, where $N$ is the total number of segments in the chain and $\Theta$ is the angle between the long axis of the segment $\boldsymbol{a}$ and the director $\boldsymbol{n}$. The function $Z(\Theta, N)$ satisfies the following differential equation which is mathematically equivalent to the equation of rotational diffusion in an external field: ${ }^{31,35}$

$$
\left(\frac{\partial}{\partial N}+U_{\mathrm{MF}}(\Theta)-D \nabla_{\Theta}^{2}\right) Z(\Theta, N)=0,
$$

where $U_{\mathrm{MF}}(\Theta)$ is the mean-field potential of the segments of the main chain. The solution of this equation can be written in the form:

$$
Z(\Theta, N)=\sum_{n} \mathrm{e}^{-\lambda_{n} N} \Psi_{n}(\Theta)
$$

where $\lambda_{n}$ and $\Psi_{n}$ are the eigenvalues and eigenfunctions of the following equation:

$$
\left(U_{\mathrm{MF}}(\Theta)-D \nabla_{\Theta}^{2}\right) \Psi_{n}(\Theta)=\lambda_{n} \Psi_{n}(\Theta)
$$

where the parameter $D$ characterizes the chain flexibility. For sufficiently long polymer chains the conformational entropy is approximately determined by the maximum eigenvalue $\lambda_{0}$ which corresponds to the eigenfunction $\Psi_{0}$. In this case the conformational entropy of the chain $-k_{\mathrm{B}} T \rho_{\mathrm{ch}} \ln Z \approx$ $-k_{\mathrm{B}} T \rho_{\mathrm{s}} \lambda_{0}$.

An explicit expression for the eigenvalue $\lambda_{0}$ can be obtained by means of the perturbation theory. ${ }^{35}$ One notes that for a vanishing mean field the eigenfunctions of this equation are the Legendre polynomials and the maximum eigenvalue is equal to zero. In the second order perturbation theory the correction to the maximum eigenvalue is determined by the following expression:

$$
\lambda_{0} \approx \frac{\left(\rho_{\mathrm{m}} J_{\mathrm{ms}} S_{\mathrm{m}}\right)^{2}}{\left(k_{\mathrm{B}} T D\right)^{2}} .
$$

Substituting the expression for $\lambda_{0}$ into that for the entropy of the main chain and then into eqn (5) for the total free energy 
and minimizing the free energy with respect to the orientational distribution function of the mesogenic groups one obtains

$$
f_{\mathrm{m}}(\boldsymbol{a})=\frac{1}{Z_{\mathrm{m}}} \exp \left[-\beta \rho_{\mathrm{m}} J_{\mathrm{mm}}^{\mathrm{eff}} S_{\mathrm{m}} P_{2}(\boldsymbol{a} \cdot \boldsymbol{n})\right]
$$

where $\beta=\left(k_{\mathrm{B}} T\right)^{-1}$ and $J_{\mathrm{mm}}^{\mathrm{eff}}=J_{\mathrm{mm}}+\left(\rho_{\mathrm{s}} J_{\mathrm{ms}}\right)^{2} /\left(k_{\mathrm{B}} T D\right)^{2}$.

Neglecting the terms which are proportional to the small orientational order parameters of the main chain segments $S_{\mathrm{s}}$ one finally obtains the following expression for the free energy of the nematic phase:

$$
\begin{aligned}
F_{\mathrm{N}}\left(\rho_{\mathrm{m}}, \rho_{\mathrm{n}}\right) / V= & k_{\mathrm{B}} T \rho_{\mathrm{n}}\left(\ln \rho_{\mathrm{n}}-1\right)+k_{\mathrm{B}} T \rho_{\mathrm{m}}\left(\ln \rho_{\mathrm{m}}-1\right) \\
& -\frac{1}{2} \rho_{\mathrm{n}}{ }^{2} U_{\mathrm{nn}}-\frac{1}{2} \rho_{\mathrm{m}}{ }^{2} U_{\mathrm{mm}}^{\mathrm{eff}}+\rho_{\mathrm{m}} \rho_{\mathrm{n}} U_{\mathrm{mn}}^{\text {eff }} \\
& -\frac{1}{2} \rho_{\mathrm{m}}{ }^{2} J_{\mathrm{mm}}^{\mathrm{eff}} S_{\mathrm{m}}{ }^{2}-k_{\mathrm{B}} T \ln Z_{\mathrm{N}}
\end{aligned}
$$

where the nematic order parameter of mesogenic groups satisfies the following self-consistent equation:

$$
S_{\mathrm{m}}=\frac{1}{Z_{\mathrm{N}}} \int_{0}^{\pi} P_{2}(\cos \gamma) \exp \left[-\beta \rho_{\mathrm{m}} J_{\mathrm{mm}}^{\mathrm{eff}} S_{\mathrm{m}} P_{2}(\cos \gamma)\right] \sin \gamma \mathrm{d} \gamma,
$$

where $\cos \gamma=(\boldsymbol{a} \cdot \boldsymbol{n})$ and where the one-particle partition function is expressed as:

$$
Z_{\mathrm{N}}=\int_{0}^{\pi} \exp \left[-\beta J_{\mathrm{mm}}^{\mathrm{eff}} S_{\mathrm{m}} P_{2}(\cos \gamma)\right] \sin \gamma \mathrm{d} \gamma
$$

In eqn (14) it is taken into account that the number density $\rho_{\mathrm{s}}$ of the segments of the main chain is directly related to the density of the side chain mesogenic groups $\rho_{\mathrm{m}}$ as $\rho_{\mathrm{s}}=q \rho_{\mathrm{m}}$, where $q$ is the average number of segments of the chain between two neighboring mesogenic groups. As a result, the free energy is expressed in terms of $\rho_{\mathrm{m}}$ and $\rho_{\mathrm{n}}$ only. One notes the effective interaction constants $U_{\mathrm{mm}}^{\text {eff }}$ and $U_{\mathrm{nm}}^{\text {eff }}$ in eqn (14) depend on the average isotropic interaction energies $U_{\mathrm{sn}}$ and $U_{\mathrm{sm}}$ between the segments of the chain and NPs and mesogenic groups, respectively, on the isotropic interaction constant $U_{\mathrm{ss}}$ between the segments of different chains and on the side-chain parameter $q$ :

$$
U_{\mathrm{mm}}^{\mathrm{eff}}=U_{\mathrm{mm}}+q^{2} U_{\mathrm{ss}}+2 q U_{\mathrm{ms}}, U_{\mathrm{mn}}^{\mathrm{eff}}=U_{\mathrm{mn}}=q U_{\mathrm{ns}} .
$$

\subsection{Coexistence of nematic and isotropic phases with different concentrations of nanoparticles}

Let us consider now the inhomogeneous system composed of the coexisting isotropic and nematic phases with different concentrations of NPs and mesogenic groups. Its total free energy can be written in the form:

$$
F_{\mathrm{NI}}^{\mathrm{sep}}=\frac{V_{\mathrm{I}}}{V} F_{\mathrm{I}}\left(\rho_{\mathrm{mI}}, \rho_{\mathrm{nI}}\right)+\frac{V_{\mathrm{N}}}{V} F_{\mathrm{N}}\left(\rho_{\mathrm{mN}}, \rho_{\mathrm{nN}}\right),
$$

where the free energy density of the isotropic phase is expressed as:

$$
\begin{aligned}
F_{\mathrm{I}}\left(\rho_{\mathrm{m}}, \rho_{\mathrm{n}}\right) / V= & k_{\mathrm{B}} T \rho_{\mathrm{n}}\left(\ln \rho_{\mathrm{n}}-1\right)+k_{\mathrm{B}} T \rho_{\mathrm{m}}\left(\ln \rho_{\mathrm{m}}-1\right) \\
& -\frac{1}{2} \rho_{\mathrm{n}}{ }^{2} U_{\mathrm{nn}}-\frac{1}{2} \rho_{\mathrm{m}}{ }^{2} U_{\mathrm{mm}}^{\mathrm{eff}}+\rho_{\mathrm{m}} \rho_{\mathrm{n}} U_{\mathrm{mn}}^{\text {eff }},
\end{aligned}
$$

where $V_{\mathrm{I}}$ and $V_{\mathrm{N}}$ are the volumes of the isotropic and the nematic phase, respectively, and where the free energy density of the nematic is given by eqn (14).

In eqn (18) $\rho_{\mathrm{mI}}$ and $\rho_{\mathrm{nI}}$ are the number densities of the mesogenic groups and NPs, respectively, in the isotropic phase, while $\rho_{\mathrm{mN}}$ and $\rho_{\mathrm{nN}}$ are the corresponding number densities in the nematic phase.

One notes that the concentrations of both NPs and mesogenic groups must generally be different in different phases. Indeed, it is known $^{\mathbf{1 5}}$ that spherical NPs dilute the nematic phase which results in a decrease of the nematic-isotropic transition temperature. As a result the free energy density of the nematic phase increases, and it may be energetically favorable for the system to phase separate into coexisting isotropic and nematic phases so that the NP concentration stays higher in the isotropic phase.

The chemical equilibrium between the nematic and the isotropic phase in our system is possible only if the chemical potentials $\mu_{\mathrm{n}}$ and $\mu_{\mathrm{m}}$ of NPs and the mesogenic groups, respectively, are the same in the two phases, and if the pressure in the two phases is also the same. For incompressible LCs only the equations for the chemical potentials are relevant: $\mu_{\mathrm{nI}}=\mu_{\mathrm{nN}}$ and $\mu_{\mathrm{mI}}=\mu_{\mathrm{mN}}$ where the chemical potentials are given by the well known general equations: $\mu_{\mathrm{i}}=\frac{\partial F}{\partial N_{\mathrm{i}}}=\frac{1}{V_{\mathrm{i}}} \frac{\partial F}{\partial \rho_{\mathrm{i}}}, \mathrm{i}=\mathrm{n}, \mathrm{m}$. Expressing the chemical potentials both in the isotropic and in the nematic phase using eqn (14) and (19) one obtains the following equations:

$$
\ln \frac{\rho_{\mathrm{mN}}}{\rho_{\mathrm{mI}}}=U_{1}\left(\rho_{\mathrm{mN}}-\rho_{\mathrm{mI}}\right)+U_{12}\left(\rho_{\mathrm{nN}}-\rho_{\mathrm{nI}}\right)+\ln Z_{\mathrm{N}}
$$

and

$$
\ln \frac{\rho_{\mathrm{nN}}}{\rho_{\mathrm{nI}}}=U_{2}\left(\rho_{\mathrm{nN}}-\rho_{\mathrm{nI}}\right)+U_{12}\left(\rho_{\mathrm{mN}}-\rho_{\mathrm{mI}}\right),
$$

where the reduced interaction constants $U_{1}=U_{\mathrm{mm}} /\left(k_{\mathrm{B}} T\right), U_{2}=$ $U_{\mathrm{nn}}^{\text {eff }} /\left(k_{\mathrm{B}} T\right)$ and $U_{12}=U_{\mathrm{nm}}^{\text {eff }} /\left(k_{\mathrm{B}} T\right)$ have been introduced.

Assuming that both phases are incompressible (that is neglecting a small density change at the transition), the number densities of NPs and mesogenic groups in each phase can be expressed in terms of the volume fraction $\phi_{\mathrm{i}}$ of NPs in the phase $\mathrm{i}=\mathrm{N}, \mathrm{I}$ :

$$
\rho_{\mathrm{ni}}=\rho_{\mathrm{n} 0} \phi_{\mathrm{i}}, \rho_{\mathrm{mi}}=\rho_{\mathrm{m} 0}\left(1-\phi_{\mathrm{i}}\right)
$$

where $\mathrm{i}=\mathrm{N}, \mathrm{I}, \rho_{\mathrm{mo}}$ is the number density of the mesogenic groups in the pure LC and $\rho_{\mathrm{n} 0}=1 / v_{\mathrm{n}}$ where $v_{\mathrm{n}}$ is the NP volume.

In this case eqn (20) and (21) can be expressed in terms of the NP volume fractions in the two phases $\phi_{\mathrm{N}}$ and $\phi_{\mathrm{I}}$ :

$$
\begin{gathered}
\ln \frac{1-\phi_{\mathrm{N}}}{1-\phi_{\mathrm{I}}}=w_{1}\left(\phi_{\mathrm{I}}-\phi_{\mathrm{N}}\right)+\ln Z_{\mathrm{N}}, \\
\ln \frac{\phi_{\mathrm{I}}}{\phi_{\mathrm{N}}}=w_{2}\left(\phi_{\mathrm{I}}-\phi_{\mathrm{N}}\right),
\end{gathered}
$$


where

$$
w_{1}=\rho_{\mathrm{m} 0} U_{1}-\rho_{\mathrm{n} 0} U_{12}, w_{2}=\rho_{\mathrm{n} 0} U_{2}-\rho_{\mathrm{m} 0} U_{12} .
$$

Solutions of eqn (23) and (24) are strongly affected by the nondimensional constants $w_{1}, w_{2}$. In particular, it can readily be shown that the phase coexistence is possible only if $w_{2}>1$. One notes also that in the polymer LC composite, studied in this paper, NPs interact very strongly with the polymer chain as a whole. As a result, the inequality $\rho_{\mathrm{n} 0} U_{12}>\rho_{\mathrm{m} 0} U_{1}$ is always satisfied and hence $w_{1}<0$. In this case the parameters $w_{1}$ and $w_{2}$ have the following physical meaning: $w_{1}$ is essentially the average interaction of all NPs in the unit volume with the polymer chains, while $w_{2}$ is the difference between the average interaction of NPs themselves and the interaction of NPs and the polymer chains. Taking into account that the volume of a typical NP (together with the volume of the attached chains) is significantly larger than that of a mesogenic group one expects that $w_{2}>0$.

Eqn (23) and (24) are to be solved with the partition function (16) and self-consistent nematic order parameter, which can only be done numerically. The procedure can be substantially simplified if one takes into account that the volume fraction of NPs in both phases is typically sufficiently small, that is $\phi_{\mathrm{I}} \ll 1$ and $\phi_{\mathrm{N}} \ll 1$. In this case eqn (23) reduces to:

$$
\left(1-w_{1}\right)\left(\phi_{\mathrm{I}}-\phi_{\mathrm{N}}\right)=\ln Z_{\mathrm{N}},
$$

One notes also that $Z_{\mathrm{N}}$ is independent of $\phi_{\mathrm{I}}$ and therefore the unknown $\phi_{\mathrm{I}}$ can be excluded from the simultaneous equations for $\phi_{\mathrm{I}} \ll 1$ and $\phi_{\mathrm{N}} \ll 1$ which results in a single equation for $\phi_{\mathrm{N}}$ :

$$
Z_{\mathrm{N}}^{w_{2} /\left(1-w_{1}\right)}=1+\frac{\ln Z_{\mathrm{N}}}{\phi_{\mathrm{N}}\left(1-w_{1}\right)}
$$

After the value of $\phi_{\mathrm{N}}$ is found, $\phi_{\mathrm{I}}$ can be evaluated as:

$$
\phi_{\mathrm{I}}=\phi_{\mathrm{N}}+\frac{\ln Z_{\mathrm{N}}}{1-w_{1}}
$$

In the experiment one normally controls the average volume fraction of NPs $\phi$ in the isotropic phase prior to the phase separation. Note that the solutions of eqn (23) and (24) are independent of $\phi$. The values of $\phi, \phi_{\mathrm{I}}$ and $\phi_{\mathrm{N}}$ enable one to determine the volumes $V_{\mathrm{I}}$ and $V_{\mathrm{N}}$ of the coexisting isotropic and nematic phases, respectively. Indeed, it follows from the conservation of the total number of NPs that $\phi V=\phi_{\mathrm{I}} V_{\mathrm{I}}+\phi_{\mathrm{N}} V_{\mathrm{N}}$ where $V=V_{\mathrm{I}}+V_{\mathrm{N}}$ is the total volume of the system. From these equations one obtains:

$$
V_{\mathrm{I}}=V \frac{\phi-\phi_{\mathrm{I}}}{\phi_{\mathrm{I}}-\phi_{\mathrm{N}}}, \quad V_{\mathrm{N}}=V \frac{\phi_{\mathrm{N}}-\phi}{\phi_{\mathrm{I}}-\phi_{\mathrm{N}}} .
$$

One can readily see from eqn (29) that the phase coexistence is possible only if $\phi_{\mathrm{N}}<\phi<\phi_{\mathrm{I}}$ as $\phi_{\mathrm{N}}<\phi_{\mathrm{I}}$. For $\phi$ outside this interval, only one phase may be stable at a given temperature.

Thermodynamically, the coexisting nematic and isotropic phases are stable only if the total free energy of the phaseseparated system is lower than the free energy of both isotropic and nematic homogeneous phases. This condition can be expressed as:

$$
\frac{F_{\mathrm{NI}}^{\mathrm{sep}}-F_{\text {hom }}}{V k_{\mathrm{B}} T}=\frac{1}{V k_{\mathrm{B}} T}\left[\frac{V_{\mathrm{I}}}{V} F_{\mathrm{I}}\left(\phi_{\mathrm{I}}\right)+\frac{V_{\mathrm{N}}}{V} F_{\mathrm{N}}\left(\phi_{\mathrm{N}}\right)-F_{\text {hom }}\right]<0,
$$

where $V_{\mathrm{I}}$ and $V_{\mathrm{N}}$ are given by eqn (29), the energy of the homogeneous state $F_{\text {hom }}$ must be taken as the lowest from $F_{\mathrm{I}}(\phi)$ and $F_{\mathrm{N}}(\phi)$, and the free energy densities $F_{\mathrm{I}}$ and $F_{\mathrm{N}}$ as functions of the variable $\phi$ are readily obtained from eqn (19), (14) and (22):

$$
\begin{gathered}
F_{\mathrm{I}}(\phi) / V k_{\mathrm{B}} T=\rho_{\mathrm{n} 0} \phi \ln \phi+\rho_{\mathrm{m} 0}(1-\phi) \ln (1-\phi)-\frac{1}{2} \rho_{\mathrm{n} 0}^{2} \phi^{2} U_{2} \\
-\frac{1}{2} \rho_{\mathrm{m} 0}{ }^{2}(1-\phi)^{2} U_{1}+\rho_{\mathrm{m} 0} \rho_{\mathrm{n} 0} \phi(1-\phi) U_{12}
\end{gathered}
$$

where $J=J_{\mathrm{mm}}^{\mathrm{eff}} / k_{\mathrm{B}} T$. When calculating the above free energies the nematic order parameter $S\left(\phi_{\mathrm{N}}\right)$ given by eqn (15) with $\rho_{\mathrm{m}}=$ $\rho_{\mathrm{mo}}\left(1-\phi_{\mathrm{N}}\right)$ and $Z_{\mathrm{N}}=Z_{\mathrm{N}}\left(\phi_{\mathrm{N}}\right)$ is to be taken for the nematic separated phase, while for the homogeneous nematic phase $S(\phi)$ is given by the same equation with $\rho_{\mathrm{m}}=\rho_{\mathrm{mo}}(1-\phi)$ and $Z_{\mathrm{N}}=Z_{\mathrm{N}}(\phi)$. Note that the evaluation of eqn (30) can be significantly simplified by taking into account that in the polymer composite materials, considered in this paper, the volume of a NP is significantly larger than that of a mesogenic group, and therefore $\rho_{\mathrm{n}} / \rho_{\mathrm{m}} \ll 1$.

\subsection{Phase diagrams of polymer nematics doped with nanoparticles}

Eqn (27), (23) and (26) have been solved numerically together with eqn (15) for the nematic order parameter for different values of temperature and the total NP volume fraction $\phi$. At each point the conclusion about the stability of either a homogeneous or the phase-separated state has been made according to the sign of eqn (30).

In numerical calculations we have used two different sets of values of parameters $w_{1}$ and $w_{2}$ which are consistent with order of magnitude estimates. As discussed in Section 2.2, parameter $w_{1}$ is mainly determined by the strong interaction between NPs and the polymer chains. Neglecting the first term in the expression for $w_{1}$ and taking into account that the interaction occurs at the surface of the NP, $\left|w_{1}\right|$ can roughly be estimated as: $\left|w_{1}\right| \sim U_{\mathrm{n}, \mathrm{ch}} V_{\text {surf }} / v$ where $U_{\mathrm{n}, \mathrm{ch}}$ is the typical value of the interaction between the oleic surface shell of a NP and the particular side chains of the copolymer, $V_{\text {surf }}$ is the volume of the surface interaction region which may have the thickness of about 1-2 $\mathrm{nm}$ and $v$ is the NP volume. Assuming that in the systems under consideration the typical interaction energy $U_{\mathrm{n}, \mathrm{ch}}$ is of the order of $10 k_{\mathrm{B}} T$ and taking into account that the typical size of a NP is about $4 \mathrm{~nm},{ }^{32}$ one obtains the estimate $\left|w_{1}\right| \sim 3-$ 10. A similar procedure reveals a slightly larger estimate for $w_{2}$ which may be 2-3 times larger than $\left|w_{1}\right|$.

For typical but not very large absolute values of parameters $w_{1}$ and $w_{2}$ the temperature-concentration phase diagram is presented in Fig. 1. For larger values of the interaction constants, which correspond to very strong interaction between 


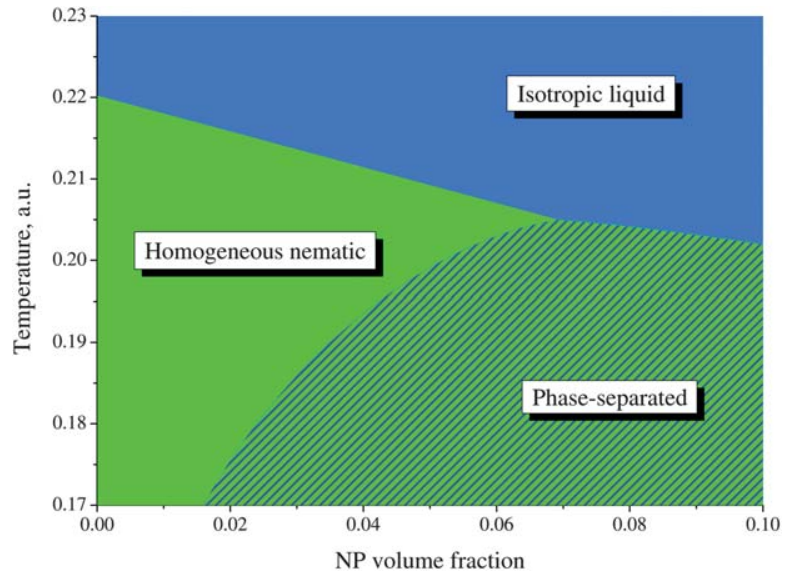

Fig. 1 Phase diagram of the composite calculated from the mean-field theory with interaction constants $w_{1}=-5$ and $w_{2}=10$.

NPs and the polymer chain, the phase diagrams are qualitatively different (see the example in Fig. 2). One can readily see that in both cases there is no phase separation at very low values of the total NP volume fraction $\phi$. In this domain, the nematicisotropic transition temperature decreases with increasing $\phi$ due to the dilution effect considered in detail in ref. 15. Above a certain critical concentration, the nematic-isotropic phase transition is accompanied by the separation between the isotropic and the nematic phase, and the two phases coexist over a significant temperature interval. In this region the transition temperature in the phase-separated state decreases more slowly than the $\mathrm{N}-\mathrm{I}$ transition temperature at low concentrations.

In the case of larger values of the interaction constants, the region of phase separation is shifted towards smaller values of $\phi$ and appears to be more narrow. This qualitative behavior is similar to the one observed experimentally as shown in the following section.

The dependence of the transition heat on the volume fraction of NPs for the same two systems is presented in Fig. 3. One notes that in the region of very low volume fractions of NPs, where the phase separation is absent, the transition heat

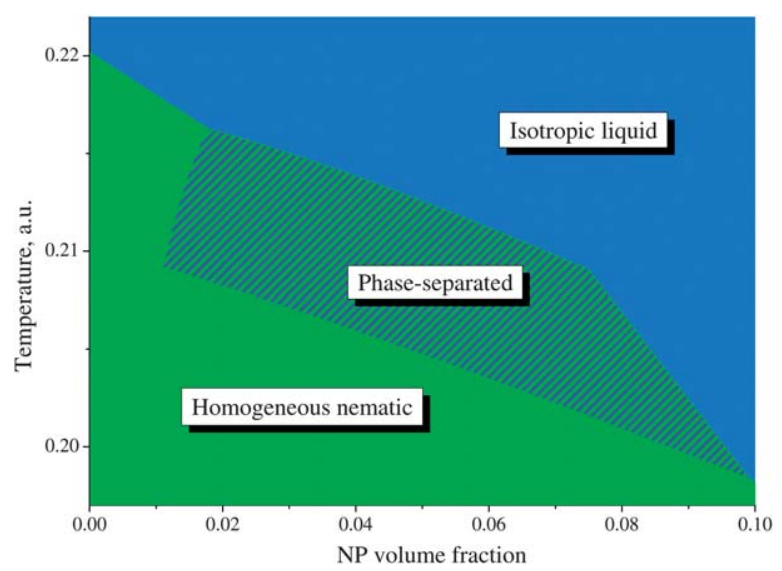

Fig. 2 The same as in Fig. 1 with $w_{1}=-10$ and $w_{2}=30$. decreases slowly with the increasing volume fraction $\phi$. In ref. 15 this decrease is related to the decrease of the volume of the nematic phase which is effectively diluted by isotropic NPs.

At higher concentrations of NPs, the system undergoes a transition from the isotropic phase into the phase-separated state. In this domain the transition heat decreases much faster with increasing NP fraction. This is related to the fact that directly below the transition temperature only a part of the system volume is in the nematic phase. As a result, the transition heat appears to be substantially smaller than that for the transition into the homogeneous nematic phase because it is proportional to the volume of the nematic phase.

Finally, temperature dependencies of the NP fractions $\phi_{\mathrm{I}}$ and $\phi_{\mathrm{N}}$ in the coexisting isotropic and nematic phases are presented in Fig. 4 for the same two systems.

One notes that the two typical phase diagrams, presented in Fig. 1 and 2, contain some rather unusual feature. Firstly, the temperature interval of the phase coexistence may be very large. In particular, in Fig. 1 this range is so broad that the system may undergo a transition into the low temperature smectic phase in the phase separated state. Secondly, the phase separation does not occur at a very low volume fraction of NPs. For large values of $w_{1}$ and $w_{2}$ the separation only occurs within a finite concentration range of NPs. One can also see in Fig. 1 that the phase separation may occur not at the N-I transition but below the homogeneous nematic phase. Moreover, in a narrow concentration region the system phase separates below the homogeneous nematic phase and then undergoes a transition into the reentrant homogeneous $\mathrm{N}$ phase at lower temperatures (see Fig. 2).

These unexpected results may be understood if one takes into consideration the fact that the phase separated system is globally stable only if three independent conditions are simultaneously satisfied. Indeed, the equilibrium NP molar fractions in the coexisting $\mathrm{N}$ and I phases are the solutions of eqn (23) and (24). Typical profiles of $\phi_{\mathrm{I}}$ and $\phi_{\mathrm{N}}$ are presented in Fig. 4, and one can readily see that the two different solutions, which correspond to the coexistence of the $\mathrm{N}$ and the I phase, exist only below some critical temperature. This critical temperature

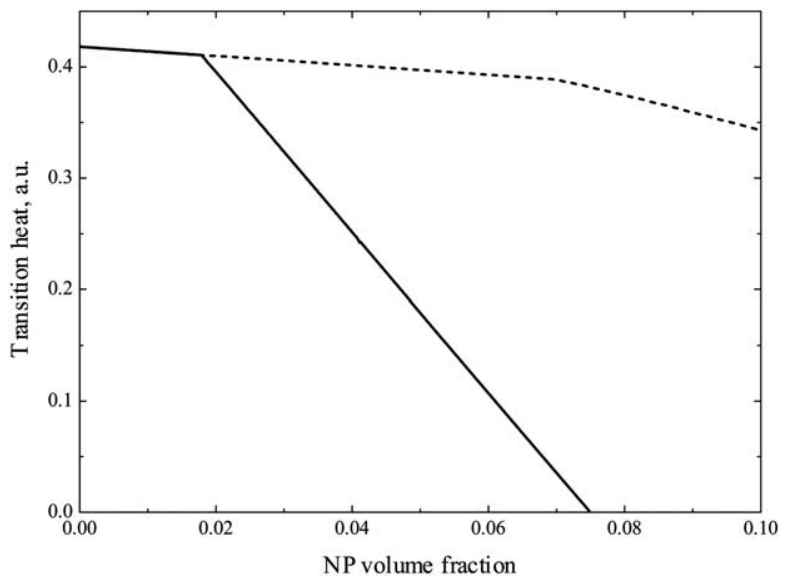

Fig. 3 Heat of the transition from the isotropic liquid phase for the composites with $w_{1}=-10, w_{2}=30$ (solid) and $w_{1}=-5, w_{2}=10$ (dashed). 


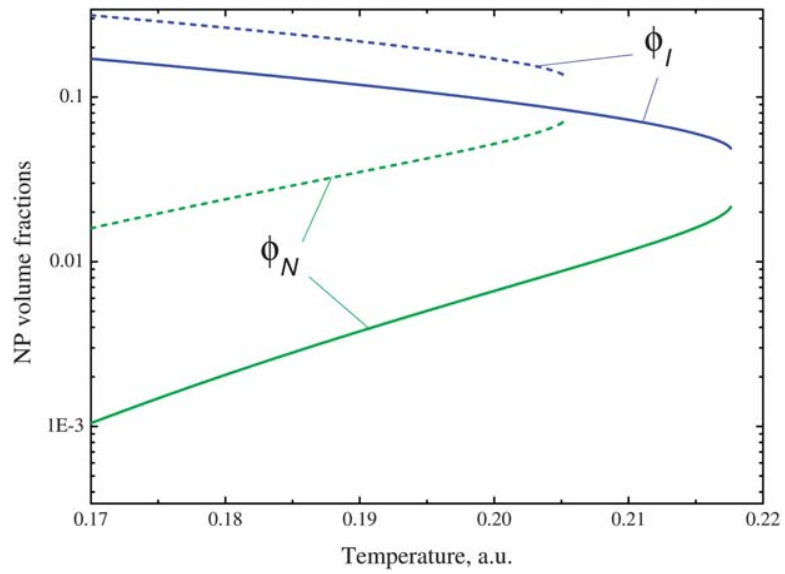

Fig. 4 Nanoparticle volume fractions in coexisting isotropic (upper curves) and nematic (lower curves) phases of the composites with $w_{1}=-10, w_{2}=30$ (solid) and $w_{1}=-5, w_{2}=10$ (dashed).

is usually higher than the actual $\mathrm{N}-\mathrm{I}$ transition temperature in the homogeneous system. Taking into account also that the two solutions exist only for nonzero values of the nematic order parameter $S$ one concludes that in this case the system undergoes a transition from the homogeneous I phase into the phase separated state with a finite difference between NP volume fractions in the coexisting phases. At the same time, the average NP molar fraction $\phi$ cannot be larger than the NP molar fraction in the coexisting $\mathrm{N}$ phase, and also it cannot be smaller than the concentration in the coexisting I phase, i.e. at a given temperature the inequality $\phi_{\mathrm{I}}<\phi<\phi_{\mathrm{N}}$ must be satisfied. If this global condition is not satisfied, the phase separation is impossible at the corresponding temperature which explains why the separation does not occur at very low and (for some values of parameters) at sufficiently high concentrations of NPs.

The phase separation may still occur at lower temperatures when $\phi$ enters the area between the solutions $\phi_{\mathrm{I}}$ and $\phi_{\mathrm{N}}$ (which broadens with decreasing temperature as shown in Fig. 4). This may occur at a temperature well below the actual N-I transition point, and this explains why the separation may occur below the homogeneous nematic phase. The solutions $\phi_{\mathrm{N}}(T)$ and $\phi_{\mathrm{I}}(T)$ have a tendency towards saturation, and thus there exist average NP concentration ranges where the phase separation never occurs. In addition, it should be taken into account that the phase separated state is globally stable only if its total free energy is lower than that of both homogeneous nematic and homogeneous isotropic phase in the same volume. This is a strong condition which explains why the temperature range of the phase separated state is generally finite as the homogeneous nematic phase normally wins at sufficiently low temperatures. This also explains why the reentrant $\mathrm{N}$ phase may be stable below the finite stability range of the coexisting phases.

\section{Experimental}

Experimental studies of the phase transitions of composites containing CdSe NPs and LC matrices were carried out with a LMW LC, and LC nematic polymers synthesized in the frameworks of the current study. Below we describe the details of the polymer and NP syntheses.

\subsection{LC systems}

Monomers, 4-(6-acryloyloxyhexyloxy)benzoic acid, 4-metoxyphenyl-4'-(6-acryloyloxyhexyloxy)benzoate and 4-(6-acryloyloxyhexyloxy)cholesterol benzoate, have been synthesized and then copolymerized as described previously. ${ }^{22}$ The chemical formulae of the synthesized copolymers CP-1 (phases: glass $27^{\circ} \mathrm{C} \mathrm{LC}_{1} 71.4^{\circ} \mathrm{C} \mathrm{LC}_{2} 104.5^{\circ} \mathrm{C}$ isotropic) and CP-2 (phases: glass $33{ }^{\circ} \mathrm{C}$ cholesteric $172.7^{\circ} \mathrm{C}$ isotropic) are presented in Fig. 5a and b. LMW LC, 3-hydroxy-4-propionylphenyloxy-4-octyl benzoate having the chemical formula presented in Fig. 5c, was kindly provided by Dr Ivan I. Konstantinov (TIPS RAS). It undergoes the following phase transitions: crystal $77.6{ }^{\circ} \mathrm{C}$ nematic $87.15{ }^{\circ} \mathrm{C}$ isotropic. $^{33}$

\subsection{Nanoparticle synthesis}

CdSe NPs were synthesized in two stages including the synthesis of cadmium oleate and the reaction between cadmium oleate and selenium trioctylphosphine. Both reactions were carried out at a constant stirring and under argon flow at an elevated temperature using a silicone oil bath. CdO (3 mmol), oleic acid $(6 \mathrm{mmol})$ and octadecene $(10 \mathrm{~mL})$ were mixed in a flask with stirring and kept at $200{ }^{\circ} \mathrm{C}$ for 90 minutes. The temperature was increased up to $230{ }^{\circ} \mathrm{C}$ and $3 \mathrm{~mL}$ of selenium solution in TOP $(1 \mathrm{M})$ was injected. This reaction was carried out for $20 \mathrm{~min}$. and then the reaction mixture was rapidly cooled down to room temperature. A $3 \times$ volume of acetone-toluene mixture $(2: 1)$ was added to precipitate the NPs. The coagulated NPs were then centrifuged at $6000 \mathrm{rpm}$ for 5 minutes. The fluid phase was decanted, and the solid powder

a)
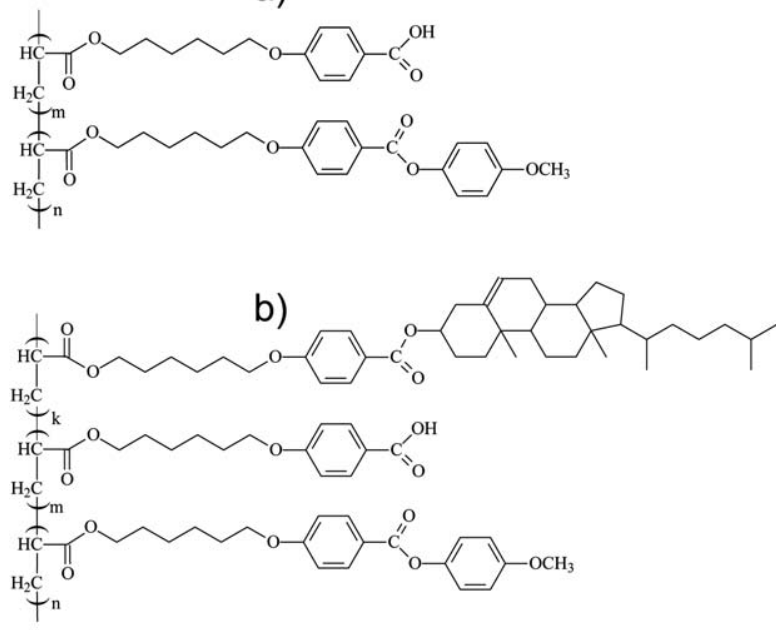

c)

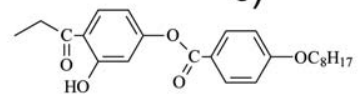

Fig. 5 The structural formulae of LC copolymers: (a) CP-1 ( $m=0.1, n=0.9)$, (b) CP-2 ( $k=0.35, m=0.05, n=0.6)$, and (c) low molecular LC. 
was washed twice with the acetone-toluene mixture and centrifuged again. The solid phase was separated, dried under argon flow and re-dissolved in hexane.

It has been shown before by the FT-IR technique that oleic acid forms a surface shell and stabilizes NPs in non-polar solvents through ionic interactions with the surface of the NPs. ${ }^{34}$ TEM images together with the photoluminescence spectra of quantum dot NPs in hexane solution were used to determine their diameter that is equal to $4.2 \mathrm{~nm} .^{32}$

\subsection{Polymer-nanoparticle composite synthesis and characterization of composites}

A special procedure for the preparation of the polymer-NP composites was developed for this study. CdSe hexane solution $\left(10 \mathrm{mg} \mathrm{mL}{ }^{-1}\right.$ ) was added drop-wise to $50 \mathrm{mg} \mathrm{mL} \mathrm{mL}^{-1}$ of the copolymer solution in methylene chloride while stirring at room temperature. The amount of CdSe solution added was calculated to provide a certain content of NPs in the final material. After stirring for 15-20 minutes the colored solution was added to $18 \mathrm{~mL}$ of hexane to precipitate the polymer-NP composite. The solid precipitate was washed with hexane and then dried overnight under vacuum at room temperature.

The composites of LMW LCs and NPs were prepared in the following way. 10-20 $\mathrm{mg}$ of the LMW LC were placed in chromatographic vials $(2 \mathrm{~mL})$ and dissolved in $0.2-0.5 \mathrm{~mL}$ of benzene. CdSe NP solution in hexane $\left(10 \mathrm{mg} \mathrm{mL}^{-1}\right)$ was added in the amount necessary to reach a certain concentration of NPS in the final blend. All solutions were transparent and looked like the homogeneous ones. Then the prepared solution was added to the DSC caps weighted in advance, and after the evaporation of the solvent the measurements of the sample mass were carried out. The initial weight of the LC and the

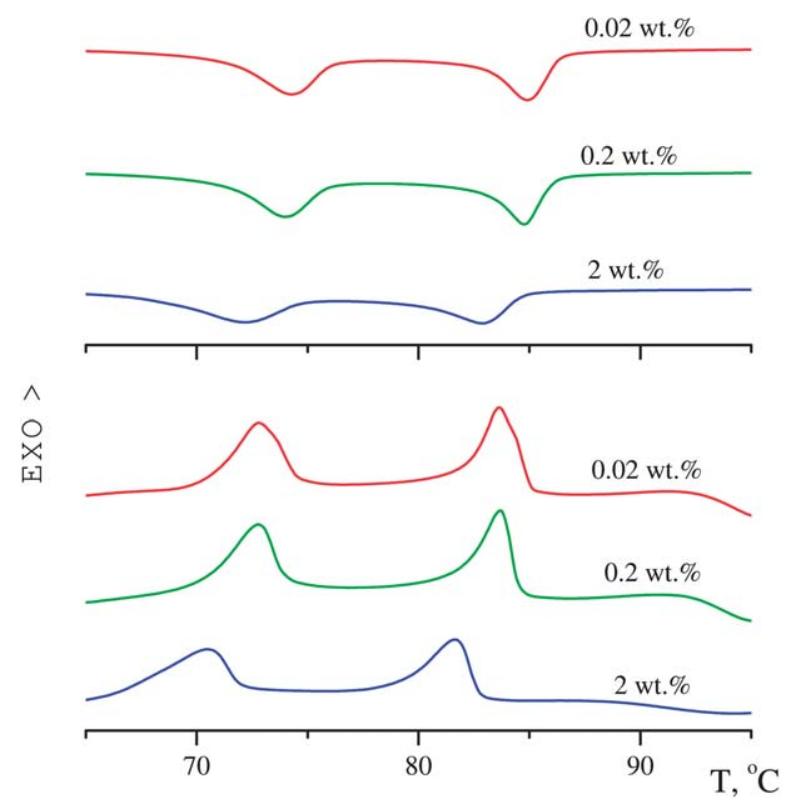

Fig. 6 Representative DSC scans of the LMW LC doped with different concentrations (shown) of NPs upon heating (upper plot) and cooling (lower plot). amount of solvent were chosen to prepare the amount of final samples for DSC measurements in the range of 3-5 $\mathrm{mg}$.

The DSC data were obtained using a differential scanning calorimeter DSC823e (Mettler Toledo) at a heating rate of $10{ }^{\circ} \mathrm{C}$ $\min ^{-1}$ in an argon atmosphere. The temperature of the tested samples was maintained with an accuracy of $0.1{ }^{\circ} \mathrm{C}$. In Fig. 6 we show representative DSC scans of the LMW LC doped with three different concentrations of CdSe NPs upon heating and cooling. The temperature and heat of the first order transitions were determined automatically by the calorimeter STARe programme. In particular, the transition temperatures correspond to the local extrema of the DSC scans (maximum/minimum heat flow). The transition heat was obtained by integrating the area between the base line and the observed line, while the integration limits were set to the zero points of the temperature derivative of the heat flow.

\subsection{Results}

The variation of the transition temperature $T_{\mathrm{NI}}$ related to the $\mathrm{N}-\mathrm{I}$ phase transition is presented in Fig. 7a, 8a and 9a. One notes that independent of the chemical structure of the nematic LC, including LMW and polymer nematics, doping of the LC matrix with NPs results in a decrease of the transition temperature $T_{\mathrm{NI}}$. The $T_{\mathrm{NI}}$ profiles can be split into several intervals of NP concentration. The initial drop of $T_{\mathrm{NI}}$ at very low contents of NPs is observed below $1 \mathrm{wt} \%$ of NPs, which is equal to $0.175 \mathrm{vol}$ $\%$. The next part of the $T_{\mathrm{NI}}$ profile is characterized by a much smaller slope and, moreover, there is a kind of a plateau of the width of about $0.2-2$ vol\% depending on the system. After such an approximately constant level region of $T_{\mathrm{NI}}$ the transition
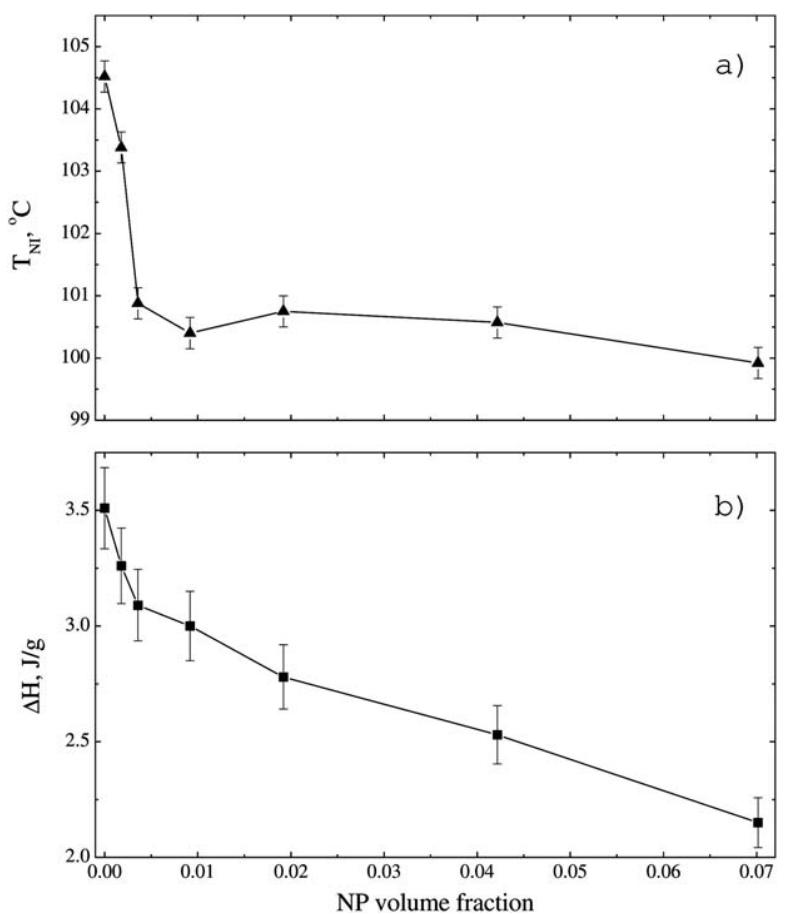

Fig. 7 Isotropic-nematic phase transition temperature (a) and enthalpy (b) as a function of nanoparticle volume fraction for composites with LC copolymer CP-1. 

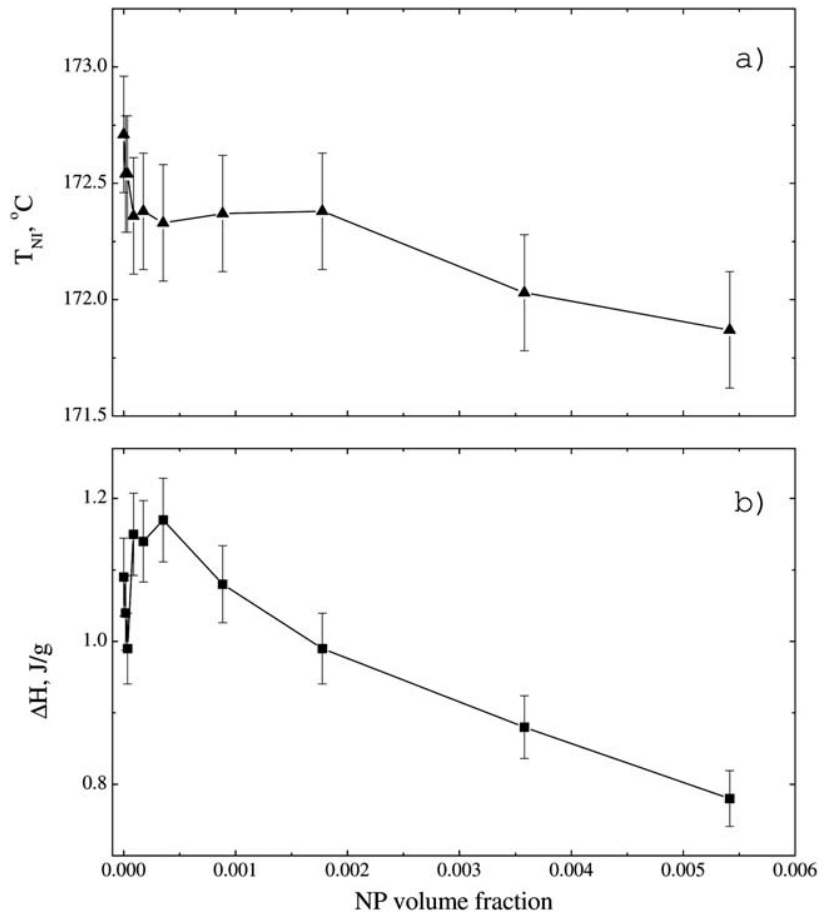

Fig. 8 The same as in Fig. 7 for composites with LC copolymer CP-2.
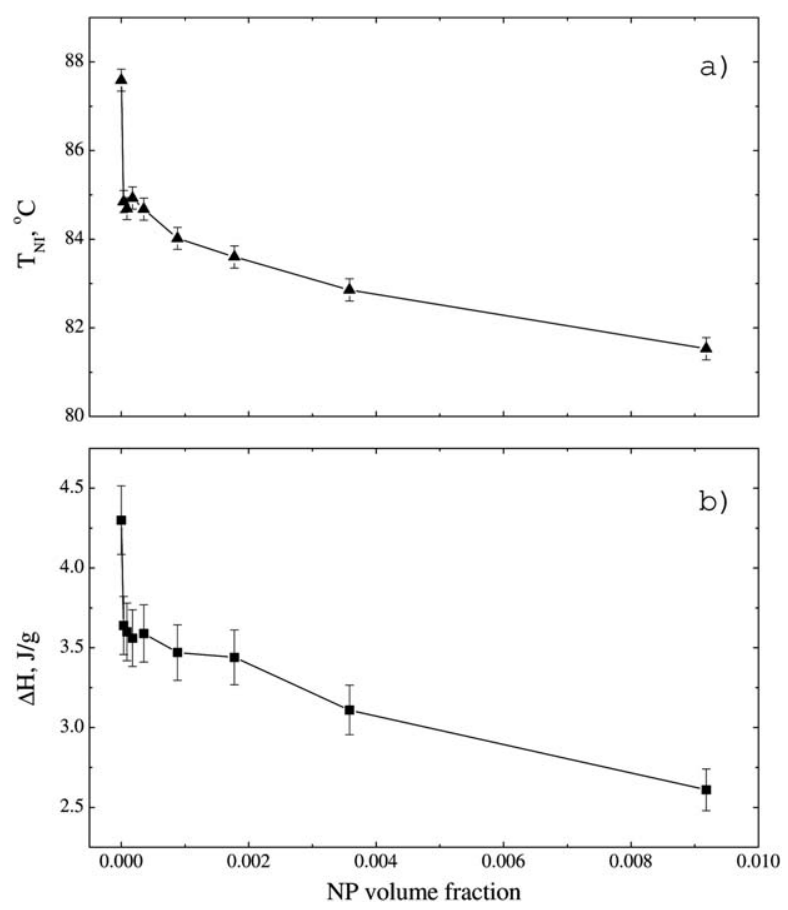

Fig. 9 The same as in Fig. 7 for composites with LMW LC.

temperature continues to decrease with the increasing concentration of NPs but with a much lower pace. The variation in the enthalpy of the phase transition is described by a similar two stage decrease in all systems although without a plateau region. In fact, the transition enthalpy decreases rather fast in the concentration region where the transition temperature is approximately constant or decreases slowly (see Fig. 7b, 8b, and 9b). One notes also that in LMW nematic composites the variation of the transition enthalpy at very low concentrations of NPs is even more complex.

\section{Discussion}

In this paper we have presented the results of both theoretical and experimental studies of the nematic-isotropic phase transition in polymers and LMW nematic LCs doped with quantum dot NPs. The molecular theory, presented in Section 2, has been developed in the mean-field approximation taking into account a possibility of the phase separation into coexisting nematic and isotropic phases induced by the transition. The NPs are assumed to be isotropic. In principle, this kind of phase separation is to be expected in binary mixtures in the vicinity of the nematic-isotropic phase transition, and it has been observed both in composite LCs containing $\mathrm{NPs}^{24}$ and in nematics doped with non-mesogenic molecules. ${ }^{26,28,35}$ However, the phase separation effects in LCs doped with NPs have never been studied systematically at different concentrations.

It should be noted that the molecular theory developed in this paper yields a number of results which are in contrast to common expectations and assumptions. Firstly, the phase separation does not necessarily occur at all concentrations of NPs, but typically the coexisting nematic and isotropic phases are stable only above a certain threshold value of the NP volume fraction which depends on the parameters of the system (see the phase diagrams in Fig. 1 and 2). There is no phase separation at very low volume fractions. Moreover, in the case illustrated by Fig. 2, the phase separation takes place only within a finite range of NP concentration, i.e. it disappears when the volume fraction of NPs exceeds the second threshold value. Secondly, the phase separation in composites does not occur only in a narrow temperature interval (as in conventional nematic mixtures) but may be present over a broad temperature range, even as broad as the nematic phase itself. Finally, in some cases (e.g. for $\phi>0.02$ in Fig. 2) the system may first undergo a transition into the homogeneous nematic phase and then separate into the nematic and the isotropic phase with different concentrations of NPs at some temperature below the $\mathrm{N}-\mathrm{I}$ transition point.

A detailed qualitative explanation of these unexpected results is presented at the end of Section 2. From the molecular point of view such an unusual behavior is related to the fact that the properties of NPs are very much different from those of typical mesogenic molecules. In particular, the interaction energy between two NPs and between a NP and a mesogenic molecule may be significantly larger than that for mesogenic molecules. The volume of a spherical NP may also be several times larger than that of an elongated mesogenic molecule of a comparable length. Thus the phase separation effects in LCs doped with NPs are expected to be significantly different from the analogous effects in LC mixtures. It is very interesting to discuss if the predictions of the theory are consistent with the experimental data presented in Section 3 of this paper.

The presented data consist of experimentally determined profiles of the $\mathrm{N}-\mathrm{I}$ transition temperature and the transition 
heat measured in the two different copolymer LCs as well as in a LMW LC doped with quantum dot NPs. One notes that in the case of both the polymer and LMW LCs, the whole domain of NP concentrations, considered experimentally, can be roughly split into two different regions which correspond to different types of variations of the transition temperature and the transition heat (see Fig. 7-9). In the first region of very small volume fractions (below 0.001) the transition temperature decreases very rapidly with the increasing NP concentration while in the second region the transition temperature is approximately constant and then decreases with the increasing concentration of NPs, but with a much smaller pace than in the first region. The decrease of the transition heat with the increasing fraction of NPs can also be split approximately into the same two regions characterized by dramatically different slopes of the profile. Thus from the general qualitative point of view the variation of the transition temperature and the transition heat is similar for the polymer and LMW LCs and should be related to some general properties of such soft matter materials.

On the other hand there exists a considerable quantitative difference both between the polymer and LMW LC composites and between binary and ternary LC copolymer composites. In particular, the width of the concentration region, in which the transition temperature varies very slowly, may differ significantly (from fractions of weight percent to 10 percent). One notes also that a very complex behavior is observed in a small concentration range at the boundary between the first and second regions which cannot be interpreted at the moment.

According to the results of the theory presented in Section 2, at very low NP concentrations there is no phase separation, and the decrease of the experimentally observed N-I transition temperature may, in principle, be explained by the dilution effect considered in detail in ref. 15. One notes that the drop of the transition temperature in this region is of the order of $1{ }^{\circ} \mathrm{C}$, which is consistent with the estimates of ref. 15. At the same time, the rate of change of the transition temperature is much larger that predicted by the theory.

Such a rapid drop of the transition temperature is observed within a very narrow range of NP concentration. As discussed above, at larger concentrations the variation of the transition temperature changes dramatically and the temperature either decreases very slowly or stays nearly constant within a certain concentration interval and then starts to decrease. Such a behavior is inconsistent with the properties of a homogeneous $\mathrm{N}-\mathrm{I}$ transition. On the other hand it can be interpreted by the molecular theory developed in this paper if one assumes that in this concentration range the system separates into the nematic and the isotropic phases with different concentrations of NPs. It follows from the theory that the temperature of the transition into such a phase separated state decreases much slower with the increasing concentration than the temperature of transition into the homogeneous nematic state.

Moreover, one can readily see in Fig. 2 that there is a discontinuous change of the slope of the transition line (in the region of transitions from the isotropic to the phase separated state) at some value of the NP concentration which is related to a different scenario of phase separation. The rate of decrease of the transition temperature increases significantly after such a discontinuity point. Similar variation of the transition temperature is observed experimentally. See, in particular, Fig. 9 where the change of the slope is strongly pronounced.

One notes also that the transition heat decreases rapidly in this concentration range including the interval where the transition temperature is nearly constant. This confirms the interpretation of our experimental data based on the presence of phase separation into coexisting isotropic and nematic phases. Indeed, the heat of transition from the isotropic to the phase separated state is smaller than that of the direct transition into the homogeneous nematic phase because in the phase separated state only a part of the system volume is in the nematic phase. The transition heat then decreases further with the increasing fraction of NPs due to a decrease of the volume occupied by the nematic phase.

It should be noted that these experimental data, which seem to be rather interesting on their own, do not, of course, present direct evidence of the phase separation in the systems considered in this paper. At the same time we do not know how they could be interpreted without an assumption of the phase separation. For example, DSC scans presented in Fig. 6 indicate that the unusual variation of the transition temperature cannot be explained by a hysteresis. In particular a correlation between the unusual variation of the transition temperature and the unusual profile of the transition heat is consistent with our assumption but it seems to be very difficult to propose an alternative explanation. A more detailed experimental study of such systems using other experimental techniques is required to clarify the situation. In general, our theory indicates that at least some of the unexpected phase separation effects should also be present in other nematic LCs doped with various strongly interacting NPs. The corresponding experimental studies may yield interesting results.

The molecular theory presented in this paper is based on a standard assumption that the coexisting phases occupy sufficiently large volumes and hence the energy of the interfacial region is negligibly small compared with the bulk energy. At the same time the energy of the boundary layer between the $\mathrm{N}$ and I phases is very important in the description of the droplets of the nematic phase in the isotropic liquid which appear close to the transition point. The critical size of such a droplet is determined by the balance between surface and bulk energies and defines the corresponding length scale which is finite. These droplets, however, are not thermodynamically stable (at least in ideal systems), and as a result the surface energy does not affect the equilibrium transition temperature. One notes, however, that this common assumption may be inconsistent if the NPs have a tendency to concentrate in the boundary region which leads to a significant increase of the interfacial energy and stabilization of the droplets. It has been shown in the past that such a stabilization occurs in nematics doped with glass spheres, ${ }^{36}$ and related effects are also present in nematic and smectic LCs doped with coated gold NPs. ${ }^{37,38}$ The latter recent papers contain also very clear demonstration of the existence of droplet networks stabilized by NPs concentrated at the boundaries. 
In polymer composites studied in this paper the surface shell of the NPs interacts very strongly with particular side chains of the copolymer macromolecule. This interaction is at least several times stronger than that between typical mesogenic groups and hence any strong inhomogeneous distribution of NPs, including the localization at the boundaries, seems to be energetically unfavorable. However, this interesting type of bulk-surface separation, which is more likely to occur in the case of weaker interaction between NPs and mesogenic molecules, definitely deserves a separate theoretical study.

The variation of the transition temperature may also be affected by a possible aggregation of NPs and, in particular, by the formation of dimers. A dimer, composed of two NPs, is sufficiently anisotropic and the corresponding anisotropic interaction between the dimers and the mesogenic molecules is expected to contribute to the stabilization of the nematic phase which should lead to an increase of the N-I transition temperature. The formation of dimers and chains of NPs has not been taken into account in the mean-field molecular theory developed in this paper. These aggregation effects will be considered in detail in our future publication.

\section{Acknowledgements}

This work was supported by the Ministry of Education and Science of Russian Federation (State Contract no. 14.740.11.0897 and Agreement no. 8509).

\section{References}

1 H. Qi, B. Kinkead and T. Hegmann, Proc. SPIE, 2008, 6911, 691106.

2 H. Qi and T. Hegmann, J. Mater. Chem., 2006, 16, 4197.

3 Y. Shiraishi, N. Toshima, K. Maeds, H. Yoshikawa, J. Xu and

S. Kobayashi, Appl. Phys. Lett., 2002, 81, 2845.

4 S. Kobayashi and N. Toshima, Inf. Disp., 2007, 23, 26.

5 H. Yoshida, K. Kawamoto, H. Kubo, T. Tsuda, A. Fujii, S. Kuwabata and M. Ozaki, Adv. Mater., 2010, 22, 622.

6 S. Kaur, S. P. Singh, A. M. Biradar, A. Choudhary and K. Sreeniva, Appl. Phys. Lett., 2007, 91, 023120.

7 A. Kumar, J. Prakash, D. S. Mehta, A. M. Biradar and W. Haase, Appl. Phys. Lett., 2009, 95, 023117.

8 O. Buchnev, A. Dyadyusha, M. Kaczmarek, V. Reshetnyak and Yu. Reznikov, J. Opt. Soc. Am. B, 2007, 24, 1512.

9 Yu. Reznikov, O. Buchnev, O. Tereshchenko, V. Reshetnyak, A. Glushchenko and J. West, Appl. Phys. Lett., 2003, 82, 1917.

$10 \mathrm{~F}$. Li, O. Buchnev, C. I. Cheon, A. Glushchenko, V. Reshetnyak, Yu. Reznikov, T. J. Sluckin and J. L. West, Phys. Rev. Lett., 2006, 97, 147801; F. Li, O. Buchnev, C. I. Cheon, A. Glushchenko, V. Reshetnyak, Yu. Reznikov, T. J. Sluckin and J. L. West, Phys. Rev. Lett., 2007, 99, 219901.

11 E. B. Barmatov, D. A. Pebalk and M. V. Barmatova, Liq. Cryst., 2006, 33, 1059.

12 P. Kopcanskya, et al., Acta Phys. Pol., A, 2010, 118, 988.

13 G. Sinha, C. Glorieux and J. Thoen, Phys. Rev. E: Stat., Nonlinear, Soft Matter Phys., 2004, 69, 031707.
14 T. Bellini, M. Buscagli, C. Chiccoli, F. Mantegazza, P. Pasini and C. Zannoni, Phys. Rev. Lett., 2000, 31, 1008.

15 M. V. Gorkunov and M. A. Osipov, Soft Matter, 2011, 7, 4348.

16 H. Duran, B. Gazdecki, A. Yamashita and T. Kyu, Liq. Cryst., 2005, 32, 815.

17 P. Kopcansky, N. Tomasovicova, M. Koneracka, M. Timko, V. Zavisova, A. Dzarova, J. Jadzyn, E. Beaugnon and X. Chaud, Int. J. Thermophys., 2011, 32, 807.

18 I. Vecchi, A. Arcioni, C. Bacchiocchi, G. Tiberio, P. Zanirato and C. Zannoni, Mol. Cryst. Liq. Cryst., 2007, 465, 271-281.

19 L. M. Lopatina and J. R. Selinger, Phys. Rev. E: Stat., Nonlinear, Soft Matter Phys., 2011, 84, 041703.

20 G. A. Shandryuk, E. V. Matukhina, R. B. Vasilev, A. Rebrov, G. N. Bondarenko, A. S. Merekalov, A. M. Gas'kov and R. V. Tal'roze, Macromolecules, 2008, 41, 2178.

21 G. I. Tselikov, V. Y. Timoshenko, Y. Plenge, E. Ruehl, A. V. Shatalova, G. A. Shandryuk, A. S. Merekalov and R. V. Talroze, Semiconductors, 2013, 47, in press.

22 A. M. Shatalova, I. Y. Kutergina, Ya. I. Derikov, G. A. Shandryuk and R. V. Talroze, Polymer Science Ser. A, 2012, 54, 12.

23 H. Qi and T. Hegmann, J. Mater. Chem., 2006, 16, 4197.

24 J. Yamamoto and H. Tanaka, Nature, 2001, 409, 321.

25 H. Yoshida, Y. Tanaka, K. Kawamoto, H. Kubo, T. Tsuda, A. Fujii, S. Kuwabata, H. Kikuchi and M. Ozaki, Appl. Phys. Express, 2009, 2, 121501.

26 G. W. Gray, Molecular structure and the properties of liquid crystals, Academic, New York, 1962.

27 H. T. Peterson and D. E. Martire, Mol. Cryst. Liq. Cryst., 1974, 25, 89.

28 R. L. Humphries and G. R. Luckhurst, Proc. R. Soc. London, Ser. A, 1976, 352, 41.

29 A. Matsuyama and R. Hirashima, J. Chem. Phys., 2008, 128, 044907.

30 A. Matsuyama, J. Chem. Phys., 2009, 131, 204904.

31 I. M. Lifshitz, A. Yu. Grosberg and A. R. Khokhlov, Rev. Mod. Phys., 1978, 50, 683.

32 A. S. Merekalov, G. A. Shandryuk, R. B. Vasil'ev, V. I. Bykov, G. N. Bondarenko, A. M. Shatalova, O. A. Otmakhova and R. V. Tal'roze, Polymer Science Ser. B, 2011, 53, 553.

33 A. Wiegeleben, D. Demus and I. I. Konstantinoff, Z. Chem., 1983, 23, 339340.

34 G. A. Shandryuk, A. V. Rebrov, R. B. Vasil'ev, S. G. Dorofeev, A. S. Merekalov, A. M. Gas'kov and R. V. Talroze, Polymer Science Ser. B, 2005, 47, 266.

35 X. J. Wang and M. Warner, J. Phys. A: Math. Gen., 1986, 19, 2215.

36 V. J. Anderson, E. M Terentjev, S. P Meeker, J. Crain and W. C. K. Poon, Eur. Phys. J. E, 2011, 4, 11.

37 J. Milette, S. J. Cowling, V. Toader, C. Lavigne, I. M. Saez, R. B. Lennox, J. W. Goodby and L. Reven, Soft Matter, 2012, 8, 173.

38 J. Milette, S. Relaix, C. Lavigne, V. Toader, S. J. Cowling, I. M. Saez, R. B. Lennox, J. W. Goodby and L. Reven, Soft Matter, 2012, 8, 6593. 